\title{
Triple Positive Antiphospholipid Syndrome in a Patient with Rectosigmoid Colon Cancer- a Rare Occurrence
}

\author{
Pour M*, Adelodun A, Omore I and Simon K \\ Department of Internal Medicine, Harlem Hospital \\ Center, USA \\ *Corresponding author: Mohammad Pour, \\ Department of Internal Medicine, Harlem Hospital \\ Center, New York, USA
}

Received: April 26, 2021; Accepted: May 26, 2021; Published: J une 02, 2021

\section{Backgrround}

Antiphospholipid Syndrome (APS) is a rare autoimmune disease characterized clinically by systemic arterial, venous, and small vessel thromboses together with pregnancy-related morbid events such as miscarriage and fetal death [1]. This syndrome is associated with the presence of autoantibodies directed against anionic phospholipids or protein-phospholipid complexes. There are several autoantibodies implicated, but there are only three well-validated antibodies in the current revised APS classification (Sydney criteria), namely the AntiCardiolipin Antibodies (aCL), anti- $\beta 2$-glycoprotein I antibodies (anti- $\beta 2-$ GPI), and Lupus Anticoagulant (LA). APS is the primary disorder when it is not associated with another autoimmune disease and is a secondary disorder when it is, such as with Systemic Lupus Erythematosus (SLE) [2]. The association between APS and cancer has been the subject of recent investigation for several years, with many reports demonstrating elevated levels of antiphospholipid antibodies (aPL) in several malignancies: lymphomas, leukemias and solid tumors of lung, kidney, stomach, liver, ovary and breast $[3,4]$. The presence of aPL in patients with solid tumors increases the risk of thromboembolism [4]. Case reports have also confirmed that the most common solid malignancies associated with APS were kidney, breast, ovarian, and lung cancers [3]. Some reports have also demonstrated an association between gastrointestinal cancers, such as gastric signet ring cell adenocarcinoma and aPL [5]. A previous study showed that moderate-to-high levels of antiphospholipid antibodies were present in a sample of 53 patients with positive CEA and CA19-9 tumor markers, suggesting a possible association with gastrointestinal tumors [6]. Here, we report the rare case of a patient with APS in the presence of invasive adenocarcinoma of the rectosigmoid colon.

\section{Case Presentation}

A 55-year-old man presented to our hospital with a fourday history of nonproductive cough and dyspnea on exertion. He reported that three months before presentation, he began to notice occasional blood in his stool, which progressed to dark red blood with every bowel movement. He also endorsed fatigue and yellowing of his skin. The patient had a thromboembolic stroke three months prior and was initiated on anticoagulation. He was aPL positive for all three APS antibodies (triple positive aPL) and met the Sydney criteria (LA,

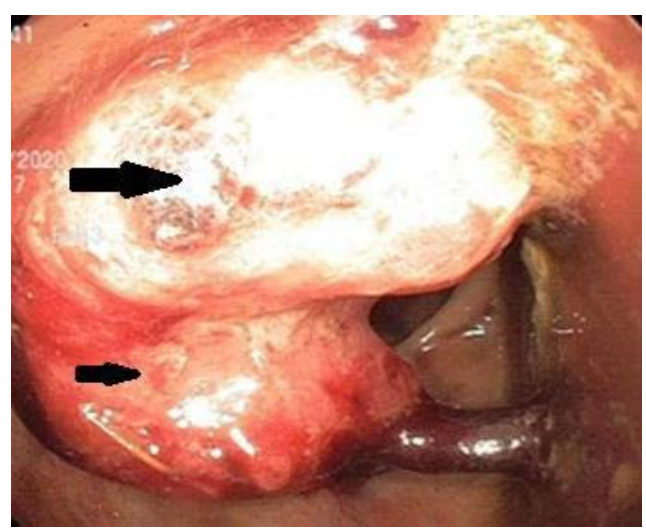

Figure 1: Rectosigmoid mass as seen on colonoscopy. Arrows indicate the malignant neoplasm, with the larger arrow indicating the part of the neoplasm directly extending from the colonic wall and the portion indicated by the smaller arrow demonstrating attachment to a peduncle. No bleeding was present. Biopsies were taken with cold forceps for histology and revealed an invasive adenocarcinoma.

$\mathrm{aCL}$, and anti- $\beta 2-\mathrm{GPI})$ on laboratory tests at that time.

On presentation, vitals were with normal limits. Abdominal examination was remarkable for epigastric tenderness without rebound or guarding. A digital rectal exam showed bright red blood on the examining glove.

Labs were significant for microcytic, hypochromic anemia with a hemoglobin of $4.0 \mathrm{~g} / \mathrm{dL}(14.0-18.0 \mathrm{~g} / \mathrm{dL})$ and a platelet count was $130,000 / \mathrm{uL}(150,000-450,000 / \mathrm{uL})$. Chest $\mathrm{x}$-ray showed an enlarged cardiac silhouette and pulmonary vascular congestion with cephalization.

The patient received two units of packed red blood cells. Esophagogastroduodenoscopy demonstrated a small hiatal hernia and $\mathrm{H}$. pylori-positive chronic active gastritis. Colonoscopy showed a partially obstructing tumor in the distal sigmoid colon that was biopsied (Figure 1). The biopsy showed invasive adenocarcinoma of the rectosigmoid colon (Figure 2). Testing for tumor markers revealed normal levels of CEA, CA-19-9, and CA-125. His rheumatoid factor was normal, as was his ANA, C3, C4, and anti-dsDNA levels. The patient was subsequently transferred to a cancer center for the treatment of his rectosigmoid adenocarcinoma.
Ann Hematol Oncol - Volume 8 Issue 7 - 2021

ISSN : 2375-7965 | www.austinpublishing group.com

Pour et al. () All rights are reserved
Citation: Pour M, Adelodun A, Omore I and Simon K. Triple Positive Antiphospholipid Syndrome in a Patient with Rectosigmoid Colon Cancer- a Rare Occurrence. Ann Hematol Oncol. 2021; 8(7): 1355. 


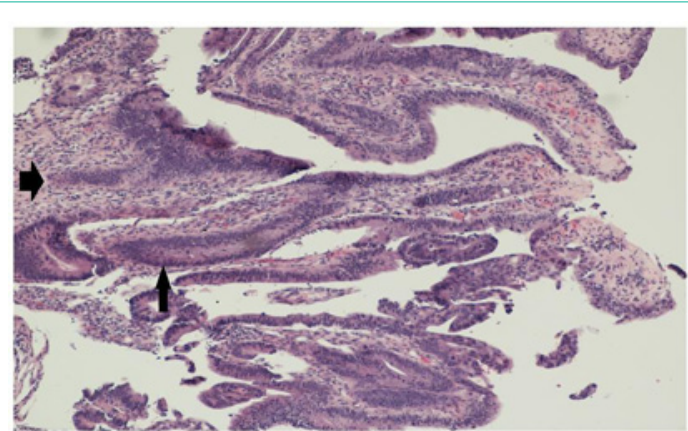

Figure 2: Histological slide demonstrating cellular atypia in the mucosal layer of the colonic villi (thin arrow). Note the dysmorphic nuclei with atypical layering of the mucosal cell layer. The malignant mucosal cells have invaded into the submucosa (thick arrow) with probable extension into the adventitia (not displayed here) typical of invasive adenocarcinoma of this kind.

\section{Discussion}

The two main clinical criteria for diagnosing APS are evidence of vascular thrombosis and pregnancy-related morbidities. Vascular thromboses include one or more blood clots -- venous, arterial, or small vessel -- in any tissue or organ. The hallmark laboratory tests for the diagnosis of APS are those that determine the presence of antiphospholipid antibodies or abnormalities in phospholipiddependent tests of coagulation. Laboratory criteria for the diagnosis of APS include the presence of lupus anticoagulant on two or more occasions at least 12 weeks apart or of anti-cardiolipin antibodies (IgG or IgM) or levels of anti-beta-2 glycoprotein I antibodies (IgG or IgM) more than the 99th percentile on two or more occasions at least 12 weeks apart. At least one clinical and one laboratory criteria are required to diagnose APS. The presence of LA itself is a strong predictor for the development of vascular thrombosis. In our case, the patient had a recent history of thromboembolic stroke in the setting of triple-positive aPLs twelve weeks prior to presentation. Our patient also presented with thrombocytopenia, one of the secondary criteria in making a diagnosis of APS. Secondary diagnostic criteria for APS include livedo reticularis, heart valve disease, thrombocytopenia, and SLE-like symptoms.

A literature review demonstrates high levels of aPLs and thromboembolic events in many solid and hematologic cancers [3]. For solid tumors, cases with APS and tumors of the kidney, lung, breast, and tumors of unknown origin were most likely associated with aPLs [1]. The pathophysiology by which aPLs generate thrombi in patients with malignancies is poorly understood. It may be that autoantibodies are a direct consequence of the tumor itself or a result of specific cancer therapies [4]. The association between aPLs and tumors may involve several mechanisms such as the production of autoantibodies reacting against tumor antigens or the secretion of aCL antibodies from tumor cells and even the production of monoclonal immunoglobulins with LA and aCL activities.

There have been reports of increased aCL and thromboembolic events in patients with colorectal cancer and a few other solid tumors [7]. In our case, a diagnosis of APS with triple-positive aPLs in the setting of rectosigmoid adenocarcinoma of the colon is a unique finding. Our patient was initiated on warfarin and referred for appropriate surgical treatment and chemoradiotherapy.

\section{Conclusion}

APS is associated with solid and hematological malignancies. The presence of aCL specifically has been reported previously in colon cancer patients. To our knowledge, this is the first reported case of a patient with triple-positive APS and rectal adenocarcinoma. We are unique because we have triple-positive APS in a male patient, and there are no previous case reports of triple-positive antibodies in colon cancer patients.

\section{References}

1. Gomez-Puerta JA, Espinosa G, Cervera R. Antiphospholipid antibodies: From general concepts to its relation with malignancies. Antibodies (Basel). 2016; 5: E18.

2. Gomez-Puerta JA, Cervera R. Diagnosis and classification of the antiphospholipid syndrome. J Autoimmun. 2014; 48: 20-25.

3. Islam MA. Antiphospholipid antibodies and antiphospholipid syndrome in cancer: Uninvited guests in troubled times. Semin Cancer Biol. 2020; 64: 108-113.

4. Tincani A, Taraborelli M, Catteneo R. Antiphospholipid antibodies, and malignancies. Autoimmun Rev. 2010; 9: 200-202.

5. Shayestehpour M, Ehsani M, Dadkhah D, Zamani B. A Case of Antiphospholipid Syndrome Following Gastric Signet Ring Cell Adenocarcinoma. Am J Case Rep. 2020; 21: e919037.

6. Pugliese L, Bernardini I, Pacifico E, et al. Antiphospholipid Antibodies in Patients with Cancer. Int J Immunopathol Pharmacol. 2006; 19: 879-888.

7. Ozguroglu M, Arun B, Erzin Y, et al. Serum cardiolipin antibodies in cancer patients with thromboembolic events. Clin Appl Thromb Hemost. 1999; 5: 181-184. 